\title{
Nitric oxide and bone: The phoenix rises again
}

\author{
Hanghang Liu' ${ }^{1}$ and Clifford J. Rosen ${ }^{2}$ \\ ${ }^{1}$ West China Hospital of Stomatology, Sichuan University, Chengdu, China. ${ }^{2}$ Maine Medical Center Research Institute, Scarborough, Maine, USA.
}

The involvement of nitric oxide (NO) in preventing bone loss has long been hypothesized, but despite decades of research the mechanisms remain obscure. In this issue of the $J \mathrm{Cl}$, Jin et al. explored NO deficiency using human cell and mouse models that lacked argininosuccinate lyase (ASL), the enzyme involved in synthesizing arginine and NO production. Osteoblasts that did not express ASL produced less NO and failed to differentiate. Notably, in the context of As/ deficiency, heterozygous deletion of caveolin 1, which normally inhibits NO synthesis, restored NO production, osteoblast differentiation, glycolysis, and bone mass. These experiments suggest that ASL regulates arginine synthesis in osteoblasts, which leads to enhanced NO production and increased glucose metabolism. After a period when research slowed, these studies, like the legendary phoenix, renew the exploration of NO in bone biology, and provide exciting translational potential.

\section{The exploration of nitric oxide in bone biology}

Almost 30 years ago, Science declared nitric oxide (NO) "molecule of the year," based on a host of breakthrough discoveries and widespread use of drugs such as nitroglycerin, nitroprusside, and sildenafil (1). Subsequent to that proclamation, an even larger, second wave of research followed to delineate the mechanism of NO actions at the molecular, cellular, and tissue level. The skeleton was one focus of those efforts. By the mid 1990s, the Osdoby and Brandi laboratories independently reported that NO synthase (NOS) was present in osteoclasts and osteoblasts, and that NO prevented osteoclast attachment and resorption (2, 3). Other studies described how NO was upregulated in osteoblasts by estrogen in a cell-autonomous manner and could drive both osteoblast proliferation and differentiation (4). An important breakthrough came with the discovery that mechanical loading of the skeleton led to a brisk NO response in osteocytes simi- lar to that induced by estrogen, and this occurred via activation of soluble guanylyl cyclase (sGC) and cGMP-dependent protein kinases (PKGs) (5).

Taken together, the strong in vitro evidence, the ease and previous widespread clinical use of NO-donor drugs, and promising observational data sustained a two-decade effort to establish organic nitrates as a preventive therapy for osteoporosis. But, the case-control, cohort, and clinical trials were either underpowered or were confounded by reverse causation $(6,7)$. More importantly, the reports from the definitive NO trial, heralded in several editorials, showing strong beneficial effects of nitroglycerin on areal and volumetric bone mineral density, and a reduction in bone resorption, were ultimately retracted because of scientific fraud in 2011 and 2017 (8, 9). Those developments, particularly the former one, basically sealed nitroglycerin's fate as a novel and cost-effective osteoporosis therapy. Unfortunately, it also slowed studies on the basic biology of NO in bone.

\section{Delated Article: https://doi.org/10.1172/JCl138935}

Conflict of interest: The authors have declared that no conflict of interest exists.

How NO drives bone formation

In this edition of the JCI, NO rises again. Jin et al. provide compelling evidence that argininosuccinate lysate (ASL) regulates arginine synthesis intracellularly in osteoblasts, which leads to enhanced NO production with subsequent upregulation of glycolytic capacity in bone-forming cells (ref. 10 and Figure 1). This aspect of the report alone provides a unique mechanism that explains how NO drives bone formation, and places the anabolic target of this molecule, i.e., glycolysis, in the same realm as parathyroid hormone effects on osteoblasts. In addition, the authors demonstrate elegantly, with induced pluripotent stem cells (iPSCs) from a single patient with ASL deficiency, that heterozygous isogenic iPSCs, through homologous recombination with a helper adenovirus, could rescue the autosomal recessive impairment in mineralization and bone formation of ASL-deficient cells (10).

Other important insights emerge from this report and add to our understanding of the complexities surrounding osteoblast differentiation as well as providing a framework for approaches to treat osteoporosis. Jin et al. used distinctive genetic models, such as the hypomorphic ASL-deficient ( $\left.A s^{\text {Neo/Neo }}\right)$ mouse, the caveolin 1 heterozygote $\left(\mathrm{Cav1}^{+/-}\right)$, and the osteoblast-specific conditional deletion of ASL to test precisely how NO regulates osteoblast differentiation (10). Those models linked NO induction and activation to both positive (arginine) and negative regulators (e.g., CAV1) that target the glycolytic pathway in osteoblasts. Notably, suppressors of NOS activity failed to rescue the cortical bone phenotype, although trabecular bone mass was restored in the cross between $\mathrm{Cav1}^{+/-}$and $A s l^{\mathrm{Neo} / \mathrm{NeO}}$ mice (10). As such, a future goal should be to define those determinants of NOS activity in osteocytes, particularly since osteocytes are the most abundant cells in the skeleton, are sensitive to mechanical loading, and could be a target for future NO-mediated antiosteoporotic agents. 


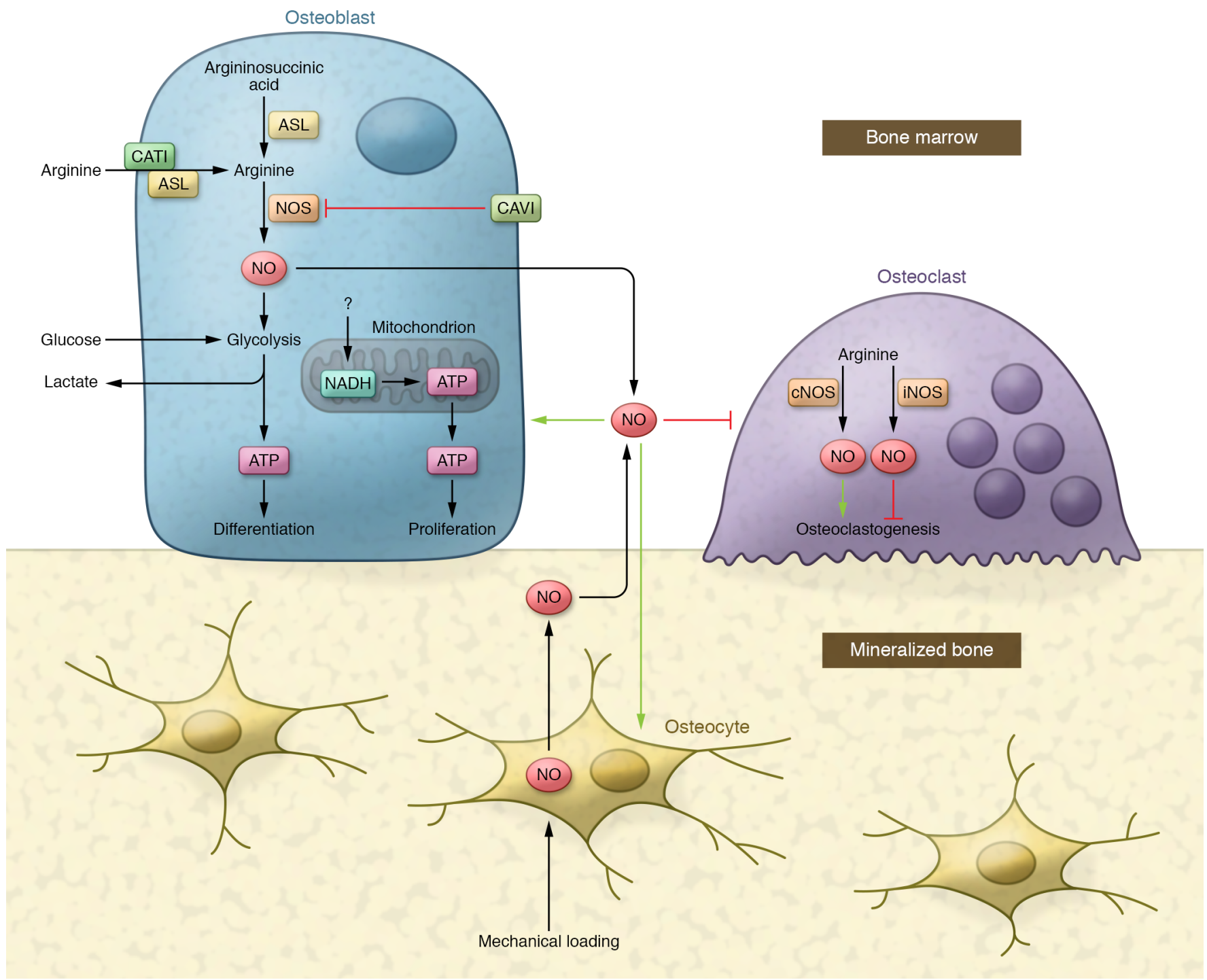

Figure 1. Model for skeletal remodeling with NO positioned at the nexus. In bone marrow, NO is produced by osteoblasts to regulate (green/red arrow) osteoblasts, osteoclasts, and osteocytes. Mechanical loading of the skeleton could also lead to a brisk NO response in osteocytes to regulate surrounded cells. The ASL enzyme generates intracellular arginine and fumarate from argininosuccinate and provides arginine for NO production in osteoblasts, while CAV1 serves as an inhibitor of NOS to decrease the generation of NO. ASL together with cationic amino acid transporter 1 (CAT1) could also help transport arginine across the membrane to increase NO production. Intracellular NO in pre- and differentiated osteoblasts promotes aerobic glycolysis, which has been considered the principal source of energy in fully differentiated osteoblasts. Exogenous NO released by osteoblasts and osteocytes prevents osteoclast attachment and resorption. However, NO generated by osteoclasts has dual effects on osteoclasts, depending on constitutive or inducible NOS activity.

\section{Addressing skeletal biology challenges}

Beyond the translational implications of NO-targeted therapeutics, the work from Jin et al. (10) underlies two ongoing challenges in skeletal biology: (a) the difficulty in defining the relative magnitude of cell-autonomous versus non-cell-autonomous determinants of osteoblast differentiation, particularly in light of the strong dose dependency of secretory factors, such as NO (11); and (b) the trouble assessing the relative balance and type of substrate utilization during osteoblast maturation (12). With respect to the former, Jin et al. created an osteocalcin-Cre targeted deletion of Asl that showed very low trabecular bone mass and markedly impaired bone formation but preserved cortical thickness (10). In contrast, the human cross-sectional study, despite including individuals with a homozygous ASL deficiency, revealed that spine bone mineral density was low in only a few individuals (10). Interestingly, those modest skeletal phenotypes actually are consistent with the generally negative outcomes from clinical nitrate trials for the prevention of bone loss $(6,7)$. But these data might also suggest that NO has noncell-autonomous effects on other tissues that can regulate bone remodeling, thereby balancing potentially adverse cell-autonomous effects from the ASL-deficient state. For example, NO can impact the CNS to drive sympathetic tone or target other cell types within the marrow niche, complicating interpretation of the skeletal phenotype (ref. 13 and Figure 1). In that 
same vein, NO can have dual effects on osteoclasts, depending on constitutive or inducible NOS activity (14). IFN- $\gamma$ induction of NOS by cells in the marrow leads to suppression of osteoclastogenesis, whereas constitutive NOS activity stimulates bone resorption (15). Furthermore, granulocyte colony-stimulating factor, in the hematopoietic niche, induces neutrophils to produce NO, which in turn can inhibit osteoblast differentiation (16). Hence, to better appreciate the full actions of $\mathrm{NO}$ in the bone marrow niche, approaches such as three-dimensional organoids, better ex vivo imaging techniques, and specialized lineage tracing methods will be required.

The second challenge to fully understanding bone formation lies in the choice of substrate utilization by the differentiating osteoblast. In general, fuel sources available to stromal cells as they progress down the osteogenic pathway include glucose, glutamate, and fatty acids. Seminal work from the Long group demonstrated that aerobic glycolysis is the principal source of energy (i.e., $80 \%$ of the ATP is generated by glycolysis) for fully differentiated osteoblasts (17). There is debate about the preeminent role of glutamate, a precursor component for the Krebs cycle, as a fuel source in normal osteoblasts, but less uncertainty exists about the preference for mitochondrial respiration over glycolysis in preosteoblasts and stromal cells. The work from Jin et al. showed that glycolysis was suppressed in the $A s l^{\mathrm{Neo} / \mathrm{Neo}}$ differentiating osteoblasts, and this suppression was rescued either by adding back SNAP (an NO donor) or by derepressing NOS inhibition by CAV1, using the heterozygous $\mathrm{CavI}^{+/-}$mouse crossed to the $A s l^{\mathrm{Neo} / \mathrm{Neo}}$ hypomorph (10). Indeed, these data fit the current paradigm that normal differentiating osteoblasts, whether stimulated by parathyroid hormone or Wnts, upregulate glycolysis and suppress mitochondrial respiration (18). In fact, even though only two ATP molecules are produced for every glucose entering the system, compared with 36 ATP molecules/glucose from fatty acid oxidation, the speed of glucose utilization and the employment of the malate-aspartate shuttle from pyruvate to prolong glycolysis while reducing mitochondrial oxidation serves to enhance the overall efficiency of the working osteoblast (19). However, osteoblast bioenergetics is temporally specific and depends on its workload (e.g., collagen formation), and as such preosteoblasts primarily rely on oxidative phosphorylation for their energy during proliferation (20). In vitro, a switch to glycolysis occurs sometime between three and four days during osteogenic differentiation, although it is unclear whether that timing is the same in vivo and what signal causes that switch (20). Therefore, it will be important to understand both what happens early in the Asl-deficient preosteoblasts and the timing of the switch to glycolysis, to determine whether the reduction in osteoblast number seen in vivo is due to an energy deficit in early oxidative phosphorylation or impaired glycolysis due to greater-than-expected glucose dependency. Those studies might provide further insight into the disposition of carbon skeletons in the absence of NO and could illuminate drug targets beyond $\mathrm{NO}$ donors.

In sum, Jin et al. have reopened the exploration of NO in bone biology using a unique translational approach involving a rare mutation in a gene generally associated with urea disposition and hepatic insufficiency (10). The importance of NO in regulating glycolysis during terminal osteoblast differentiation provides opportunities to understand a former "molecule of the year" in the context of bone remodeling. Yes, the phoenix may indeed rise from the ashes of earlier studies, and its presence may lead to exciting translational innovations.

\section{Acknowledgments}

This work was supported by the Northern New England Clinical and Translational Research Network (NIH grant U54GM115516).

Address correspondence to: Clifford J. Rosen, 81 Research Drive, Scarborough, Maine 04074, USA. Email: rosenc@mmc.org.

1. Koshland DE Jr. The molecule of the year. Science. 1993;262(5142):1953.

2. Sunyer T, et al. Proinflammatory agents, IL-8 and IL-10, upregulate inducible nitric oxide synthase expression and nitric oxide production in avian osteoclast-like cells. J Cell Biochem. 1996;60(4):469-483.

3. Brandi ML, et al. Bidirectional regulation of osteoclast function by nitric oxide synthase isoforms. Proc Natl Acad Sci U S A 1995;92(7):2954-2958.

4. Johnson DL, et al. Fluid flow stimulates rapid and continuous release of nitric oxide in osteoblasts. Am J Physiol. 1996;271(1 pt 1):E205-E208.

5. Korkmaz Y, et al. Localization of the NO-cGMP signaling pathway molecules, NOS III-phosphorylation sites, ERK1/2, and Akt/PKB in osteoclasts. J Periodontol. 2004;75(8):1119-1125.

6. Wimalawansa SJ, et al. Transdermal nitroglycerin therapy may not prevent early postmenopausal bone loss. J Clin Endocrinol Metab. 2009;94(9):3356-3364.

7. Misra D, et al. Intermittent nitrate use and risk of hip fracture. Am JMed.2017;130(2):229.e15-229.e20

8. Jamal SA, et al. Retraction to: Nitrate use and changes in bone mineral density: the Canadian Multicentre Osteoporosis Study. Osteoporos Int. 2017;28(1):421.

9. Eastell R, et al. Notice of retraction: Jamal SA, et al. Effect of nitroglycerin ointment on bone density and strength in postmenopausal women: a randomized trial. JAMA. 2011;305(8):800-807.

10. Jin Z, et al. Anabolic effects of nitric oxide on osteoblast metabolism revealed by deficiency of argininosuccinate lyase. J Clin Invest. 2021;131(5):e138935.

11. Yang JY, et al. Nitric oxide-induced autophagy in MC3T3-E1 cells is associated with cytoprotection via AMPK activation. Korean J Physiol Pharmacol. 2015;19(6):507-514.

12. Motyl KJ, et al. Energy metabolism of bone. Toxicol Pathol. 2017;45(7):887-893.

13. Jalnapurkar S, et al. Microvesicles secreted by nitric oxide-primed mesenchymal stromal cells boost the engraftment potential of hematopoietic stem cells. Stem Cells. 2019;37(1):128-138.

14. Evans DM, Ralston SH. Nitric oxide and bone. J Bone Miner Res. 1996;11(3):300-305.

15. de Bruin AM, et al. Impact of interferon- $\gamma$ on hematopoiesis. Blood. 2014;124(16):2479-2486.

16. Zhao J, et al. G-CSF inhibits growths of osteoblasts and osteocytes by upregulating nitric oxide production in neutrophils. J Craniofac Surg. 2019;30(8):e776-e780.

17. Karner CM, Long F. Glucose metabolism in bone. Bone. 2018;115:2-7.

18. Chen $\mathrm{H}$, et al. Increased glycolysis mediates Wnt7b-induced bone formation. FASEB J. 2019;33(7):7810-7821.

19. Lee WC, et al. Malic enzyme couples mitochondria with aerobic glycolysis in osteoblasts. Cell Rep. 2020;32(10):108108.

20. Lee WC, et al. Energy metabolism of the osteoblast: implications for osteoporosis. Endocr Rev. 2017;38(3):255-266 